\title{
Prospects for Studying the Local Group with the Subaru Telescope
}

\author{
K. Sekiguchi \\ Subaru Telescope, National Astronomical Observatory of Japan, \\ 650 North A'ohoku Place, Hilo, Hawaii 96720, USA
}

\begin{abstract}
Subaru Telescope is an 8.3-m diameter optical-infrared newgeneration telescope under construction on Mauna Kea, Hawaii. The telescope is expected to be operational by early 1999. The current status of its construction and the plans for its instrumentation are presented. The design specifications of the telescope and its seven common user instruments are described, with emphasis on their application to studies of the Local Group.
\end{abstract}

\section{Introduction}

Subaru Telescope is a new-generation telescope being constructed by the National Astronomical Observatory of Japan (NAOJ) under the auspices of the Japanese Ministry of Education, Science, Sports and Culture. Its primary mirror, $8.3 \mathrm{~m}$ in diameter (with an effective aperture of $8.2 \mathrm{~m}$ ), is the largest monolithic mirror ever made for an optical-infrared telescope in the world. Construction of the telescope began in 1991 at the summit of Mauna Kea, Hawaii. It has four instrument stations, two Nasmyth, one Cassegrain and one prime. Interface conditions and specifications of these focal stations are described by Iye (1995). The Cassegrain focus, with a 6 arcmin field of view (FOV), will be operational in January 1999. The Nasmyth (with 4 arcmin FOV) and the prime (with 30 arcmin FOV) foci will be implemented later in 1999.

There are seven common user instruments under construction for the Subaru Telescope. They are listed in Table 1 together with their main characteristics. Detailed descriptions of these instruments can be found in the Subaru Telescope web page "http://www.naoj.org/instruments/". Introduction of these instruments to Subaru Telescope will commence during the next two years. Subaru Telescope along with its instruments will be open to the world astronomical community by the end of the year 2000 .

\section{Status and Schedule}

Construction of the telescope enclosure and telescope mount has been completed. The figuring of the primary mirror was performed at Contraves Brashear Systems in Pittsburgh, Pennsylvania USA. The primary mirror is expected to be delivered to the summit of Mauna Kea by the beginning of November 1998. The 
Table 1. Subaru Telescope Common User Instruments.

\begin{tabular}{|c|c|c|c|c|c|c|c|c|}
\hline Name* & P.I. & Focus & Modes & $\begin{array}{c}\text { Spectral } \\
\text { Range }\end{array}$ & $\begin{array}{c}\text { Spectral } \\
\text { Resolution }\end{array}$ & Field Size & $\begin{array}{l}\text { Pixel } \\
\text { Scale }\end{array}$ & Array Bize \\
\hline FOCAS & $\begin{array}{l}\text { K. Sekiguchi } \\
\text { (NAOAf) }\end{array}$ & Cassegrain & $\begin{array}{l}\text { Imaging, } \\
\text { Grism - } \\
\text { Spectroscopy, } \\
\text { Polarimetry }\end{array}$ & $\begin{array}{l}350-1,100 \\
n m\end{array}$ & $10-2,000$ & $6-6$ & $0.1^{\prime \prime}$ & $\begin{array}{l}2018 \times 4096 \\
\operatorname{CCD} \times 2\end{array}$ \\
\hline IRCS & $\begin{array}{l}\text { A. Tokunaga } \\
\text { (Univ. Hawaii) }\end{array}$ & Cassegrain & $\begin{array}{l}\text { Imaging. } \\
\text { Grism / Echelle } \\
\text { Spectroscopy }\end{array}$ & $\begin{array}{l}1,000-5,000 \\
n m\end{array}$ & $\begin{array}{l}300- \\
20,000\end{array}$ & $\begin{array}{l}23^{\prime \prime} \times 23^{\prime \prime} \\
60^{\prime \prime} \times 60^{\prime \prime}\end{array}$ & $\begin{array}{l}0.023^{\prime \prime} \\
0.060^{\prime \prime}\end{array}$ & $\begin{array}{l}1024 \times 1021 \\
\operatorname{lnSb}\end{array}$ \\
\hline CIAO & $\begin{array}{l}\text { M. 'Tamura } \\
\text { (NAO.l) }\end{array}$ & Cassegrain & \begin{tabular}{|l|} 
Imaging \\
(Coronagraphic), \\
Grism \\
\end{tabular} & $\begin{array}{l}900-5,300 \\
\mathrm{~nm}\end{array}$ & $1,000)$ & $\begin{array}{l}12^{\prime \prime} \times 12^{\prime \prime} \\
21^{\prime \prime} \times 21^{\prime \prime}\end{array}$ & $\begin{array}{l}0.012^{\prime \prime} \\
0.024^{\prime \prime}\end{array}$ & $\begin{array}{l}1024 \times 1024 \\
\text { InSb }\end{array}$ \\
\hline COMICS & \begin{tabular}{|l} 
H. Kataza \\
('Tokyo Univ.)
\end{tabular} & Cassegrain & \begin{tabular}{|l|} 
Imaging, Grating \\
Spectroscopy
\end{tabular} & $\begin{array}{l}8,000-13,000 \\
\mathrm{~nm}\end{array}$ & $2,(0 \times)$ & $12^{\prime \prime} \times 31^{\prime \prime}$ & $0.1^{\prime \prime}$ & $\begin{array}{l}320 \times 240 \\
\text { Si:As B1B x } \\
6\end{array}$ \\
\hline HDS & $\begin{array}{l}\text { N. Noguchi } \\
\text { (NAOfl) }\end{array}$ & $\begin{array}{l}\text { Nasmyth } \\
\text { (Optical) }\end{array}$ & $\begin{array}{l}\text { Echelle } \\
\text { Spectroscopy }\end{array}$ & $\begin{array}{l}300-1,000 \\
\mathrm{~nm}, 1,000 . \\
2,000 \mathrm{~nm}\end{array}$ & 100,000 & $10^{\prime \prime} \times 0.1^{\prime \prime}$ & $0.13^{\prime \prime}$ & $\begin{array}{l}2018 \times 1096 \\
\operatorname{CCD} \times 2\end{array}$ \\
\hline OHS & $\begin{array}{l}\text { T. Maihara } \\
\text { (Kyoto Univ.) }\end{array}$ & Nasmyth (IIR) & $\begin{array}{l}\text { Low Background } \\
\text { Spectroscopy }\end{array}$ & $\begin{array}{l}1,000- \\
2.000 \mathrm{~mm}\end{array}$ & $10-1,200$ & $20^{\prime \prime} \times 20^{\prime \prime}$ & $0.12^{\prime \prime}$ & $\begin{array}{l}1024 \times 1021 \\
\mathrm{HgCd} T \mathrm{Te}\end{array}$ \\
\hline $\begin{array}{l}\text { Suprime- } \\
\text { Cam }\end{array}$ & $\begin{array}{l}\text { S. Okamura } \\
\text { (Tokyo Univ.) }\end{array}$ & Prime (/Cass.) & $\begin{array}{l}\text { Wide lield } \\
\text { lmaging }\end{array}$ & $300-1,100 \mathrm{~nm}$ & & $30^{\prime} \times 20^{\prime}$ & $0.2^{m}$ & $\begin{array}{l}2018 \times 1096 \\
\operatorname{CCD} \times 10\end{array}$ \\
\hline $\begin{array}{l}\text { CIAO (Coro } \\
\text { COMICS (C } \\
\text { HDS (High } \\
\text { OHS (OH a } \\
\text { Suprime-C }\end{array}$ & $\begin{array}{l}\text { Lagraph Imager } \\
\text { Doled Mid-Infra } \\
\text { Dispersion Spec } \\
\text { rglow Suppressi } \\
\text { n (Subaru Prim }\end{array}$ & $\begin{array}{l}\text { with Adaptiv } \\
\text { red Camera a } \\
\text { trograph) } \\
\text { ion spectrogra } \\
\text { e-Focus Cam }\end{array}$ & $\begin{array}{l}\text { e Optics) } \\
\text { and Spectrometer) } \\
\text { era) }\end{array}$ & & & \multicolumn{3}{|c|}{$\begin{array}{l}: \text { (Sasaki et al. 1995) } \\
: \text { (Tokunaga et al. 1998) } \\
: \text { (Onaka et al. 1998) } \\
: \text { (Young et al. 1998) } \\
: \text { (Bell et al. 1998) } \\
: \text { (Tamura et al. 1998) } \\
: \text { (Onaka et al. 1995) } \\
: \text { (Noguchi et al. 1998) } \\
: \text { (Motohara et al. 1998) } \\
: \text { (Miyazaki et al. 1998) }\end{array}$} \\
\hline
\end{tabular}

astronomical first light of Subaru Telescope is scheduled for the end of January 1999.

By its first light, Subaru Telescope will have five test instruments at the Cassegrain focus. These are : 1) a $1024 \times 1024$ optical CCD camera, 2) the high-speed optical CCD camera (VTOS) for speckle observations, 3) the midinfrared camera (MIRTOS : Tomono \& Nishimura 1998), 4) the $1024 \times 1024$ HgCdTe IR camera (CISCO, the camera part of the OHS; cf. Motohara 1998) and 5) the Suprime-Cam.

These test instruments will be used to determine the telescope characteristics, such as the optical quality, the tracking capability, the thermal background, etc.. Also, planned are some scientific observations using these test instruments during the telescope's performance verification period (the first six months after the first light).

After the initial tests of the telescope itself, the performance verification tests for the seven common user instruments will follow. The schedule for the commissioning of these instruments is shown in Fig. 1.

\section{Subaru Telescope and Local Group Studies}

There are various ways in which Subaru Telescope will improve Local Group studies. A large aperture with adaptive optics capability in the near-infrared bands will allow us to study individual stellar components in the Local Group in more detail than is possible today. The multi-object spectrograph (i.e. FOCAS) 


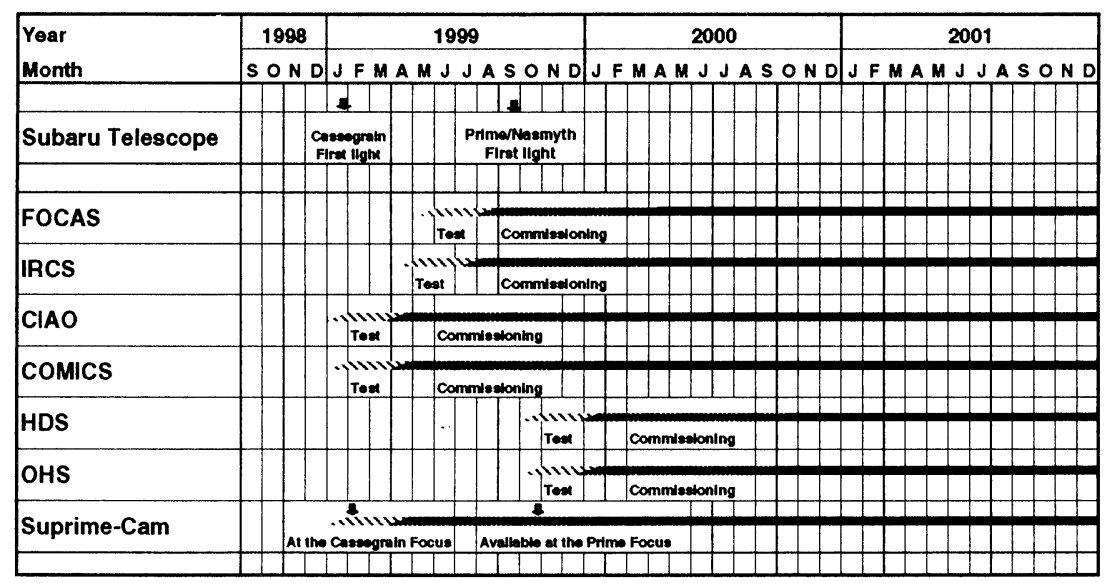

Figure 1. Subaru Telescope Instrumentation Schedule

will provide much demanded spectroscopic data for fainter objects efficiently. The wide-field capability of Subaru Telescope (i.e. Suprime-Cam) is ideal for observing extended regions of the Local Group galaxies. In the following, we briefly mention two specific projects which are planned by Subaru Telescope's instrument groups as their PV phase observations. Of course, the possibilities of using Subaru Telescope and its instruments for Local Group studies are not limited to what we mention here. A variety of other programmes are planned by the potential users. We encourage the attendees of this Symposium and the readers of these Proceedings to propose interesting programmes using the Subaru Telescope.

\section{Optical monitoring of M31 using Suprime-Cam}

Repeated observations of a $20^{\prime} \times 30^{\prime}$ field of the outer region of M31 are proposed. The aims of this programme are to identify the various types of variable stars to $V \geq 29$ and to find novae in the region. Population studies of variable stars will be undertaken. Their radial distributions will be investigated. The frequency of novae and their absolute magnitude - decay rate relation can be studied. This programme should provide a statistically meaningful number of each sub-class of variable star in M31.

\section{Optical identification of the X-ray Sources in M31}

Observations to optically identify the X-ray sources in M31, discovered by the X-ray satellites such as AXAF and XMM, are planned. High-mass X-ray binary systems can be easily identified and their spectra can be obtained. Also, Low-mass X-ray binary systems and Supersoft X-ray sources may be observed. The variability of these objects can be investigated to find out their identity. 


\section{Remarks}

By resolving the integrated light of galaxies into individual stars, Subaru Telescope will turn studies of Local Group galaxies into stellar astronomy. Physical properties of individual stars in Local Group galaxies can be compared directly with those of Galactic objects. Subaru Telescope will make observations of stellar components of the Local Group more easy but also it will provide a much larger amount of data in order to be able to discriminate between theoretical models of galactic chemical evolution and place tight constraints on the formation and the evolution of the Local Group galaxies. For stellar population studies in Local Group galaxies, the possibility to extend the absolute magnitude range of accessible stars by a factor of two or more is extremely valuable.

\section{References}

Bell, J., Douglas, J.W., Hodapp, K.-W., Robertson, L., Tokunaga, A.T., Young, T.T. 1998, in SPIE Proc., 3354-124, 1103

Iye, M. 1995, in: Scientific and Engineering Frontiers for 8 - 10m Telescopes, (eds.) M. Iye \& T. Nishimura, Tokyo: Universal Academy Press, p. 67

Motohara, K., Maihara, T., Iwamuro, F., Oya, S., Imanishi, M., Terada, H., Goto, M., Iwai, J., Tanabe, H., Tsukamoto, H., Sekiguchi, K. 1998, SPIE Proc., 3354-86, 659

Miyazaki, S., Sekiguchi, M., Imi, K., Okada, N., Nakata, F., Komiyama, Y. 1998, SPIE Proc., 3355-107, 363

Noguchi, K., Ando, H., Izumiura, H., Kawanomoto, S., Tanaka, W., Aoki, W. 1998, SPIE Proc., 3355-19, 354

Onaka, P.M., Tokunaga, A.T., Kobayashi, N., Weber, M. 1998, SPIE Proc., 3354-70, 30

Onaka, T., Kataza, H., Miyata, T., Mori, A., Tomono, D., Tanabe, T., Shibai, H., Yamashita, T., Nishimura, T. 1995, in: Scientific and Engineering Frontiers for $8-10 \mathrm{~m}$ Telescopes, (eds.) M. Iye \& T. Nishimura, Tokyo: Universal Academy Press, p. 273

Sasaki, T., Iye, M., Yamashita, T., Shibata, T., Kashikawa, N., Ohta, K., Yoshida, M., Kosugi, G., Yamada, T., Yadomaru, Y., Ozawa, T. 1995, in: Scientific and Engineering Frontiers for 8 - 10m Telescopes, (eds.) M. Iye \& T. Nishimura, Tokyo: Universal Academy Press, p. 191

Tamura, M., Suto, H., Takami, H., Itoh, Y., Ebizuka, N., Murakawa, K., Kaifu, N., Takato, N., Takeyama, N., Iye, M., Chikami, K., Oasa, Y. 1998, SPIE Proc., 3354-142, 845

Tokunaga, A.T., Kobayashi, N., Bell, J., Ching G.K., Hodapp, K.-W., Hora, J.L., Neill, D., Onaka, P.M., Rayner, J.T., Robertson, L., Warren, D.W., Weber, M., Young, T.T. 1998, SPIE Proc., 3354-35, 512

Tomono, D., Nishimura, T. 1998, SPIE Proc., 3354-130, 1150

Young, T.T., Tokunaga, A.T., Hora, J.L., Robertson, L., Kobayashi, N. 1998, SPIE Proc., 3354-71, 317 


\section{Discussion}

Marconi: Which CCDs do you plan to use for the prime-focus camera? Do you have any idea as yet about their quantum efficiency as a function of wavelength? Sekiguchi: We are planning to use the MIT Lincoln Laboratory's 3-side buttable $4096 \times 204815 \mu \mathrm{m}$ pixel CCDs. The characteristics of these are described by Miyazaki et al. (1998 SPIE, 3355, 364-374). 